\title{
ACCURATE APPROXIMATION ALGORITHM FOR TOA-BASED MAXIMUM LIKELIHOOD MOBILE LOCATION USING SEMIDEFINITE PROGRAMMING
}

\author{
${ }^{\dagger}$ K.W. Cheung, ${ }^{\ddagger \star} W .-K . M a$, and ${ }^{\dagger} H . C$. So \\ $\dagger$ Department of Computer Engineering and Information Technology, \\ City University of Hong Kong, Hong Kong \\ ${ }^{\ddagger}$ Department of Electronic Engineering, The Chinese University of Hong Kong, \\ Shatin, N.T., Hong Kong. \\ E-mail: ithcso@cityu.edu.hk,w.ma@ee.mu.oz.au, gary.cheung@student.cityu.edu.hk
}

\begin{abstract}
The techniques of using wireless cellular networks to locate mobile stations have recently received considerable interest. This paper addresses the problem of maximumlikelihood (ML) location estimation using (uplink) time-ofarrival (TOA) measurements. Under the standard assumption of Gaussian TOA measurement errors, ML location estimation is a nonconvex optimization problem in which the presence of local minima makes the search of the globally optimal solution hard. To circumvent this difficulty, we propose to approximate the ML problem by relaxing it to a convex optimization problem, namely semidefinite programming. Simulation results indicate that this semidefinite relaxation location estimator provides mean square position error performance close to the Cramér-Rao lower bound for a wide range of TOA measurement error levels.
\end{abstract}

\section{INTRODUCTION}

The capability of accurately positioning a mobile station will be one of the essential features in the existing and future wireless cellular communication systems. Mobile positioning enables many applications such as location of an emergency call [1], intelligent transport systems, and interactive map consultation [2]-[4], and for this reason it has recently received much attention.

Mobile positioning can be achieved by using the existing global positioning system (GPS). However, employing the GPS for mobile positioning would mean addition of hardware in the mobile station (MS), which is not a cost effective approach. Presently, much emphasis has been placed on utilizing the base stations (BSs) to locate the MS. The basic principle of the BS approach [4]-[6] is to use multiple BSs to intercept the MS signal for extracting location-bearing parameters. One commonly used location-bearing parameter is the time-of-arrival (TOA); i.e., the one-way signal propagation time from the MS to a BS. Assuming line-of-sight propagation and zero TOA measurement error, the TOA of each BS provides a circle centered at the BS on which the MS must lie. With three or more BSs, the MS location can

This work was partially supported by a grant from the Research Grants Council of the Hong Kong Special Administrative Region, China (Project No. CityU 1119/01E).

${ }^{\star}$ Dr. Ma is now with the Department of Electrical and Electronic Engineering, the University of Melbourne, Australia. be uniquely determined by finding the intersecting point of the TOA-derived circles.

In practice, the TOA measurements may contain error due to various factors such as noise disturbance in the received signal. Under such circumstances maximumlikelihood (ML) estimation is a powerful method of estimating the MS location. Based on the standard assumption of Gaussian distributed TOA measurement error, the ML location estimate is the globally optimal solution of a nonconvex optimization problem [7]. Traditionally, Taylor series approximation is applied to the ML problem to attempt to find the solution of the ML optimization problem in an iterative manner. This idea results in the so-called linearized least squares (LLS) location estimator [6]-[7]. Like many iterative minimization techniques, the location accuracy of the LLS estimator depends much on the initial guess of the MS location. In particular, poor initialization is likely to result in convergence to local minima. In this paper, we propose a different approach of approximating the ML problem, namely semidefinite relaxation [10]-[12]. We consider relaxing the ML problem to construct a suboptimal ML but simpler optimization problem. The resultant relaxed ML problem is a semidefinite program (SDP) [10], which is not only convex but has its optimization algorithms well developed in the optimization context. Hence, the SDR approximate ML location estimator can be effectively implemented by employing certain readily available SDP solvers.

The organization of this paper is as follows. In Section 2, the TOA-based location estimation problem is formulated and the ML method is introduced. In Section 3, the semidefinite relaxation (SDR) method for approximate ML location estimation is developed. Simulation results are presented in Section 4 where we evaluate the location estimation performance of the proposed SDR location estimator. Finally, conclusions are drawn in Section 5.

\section{PROBLEM STATEMENT}

In this section we describe the TOA-based mobile location problem and the associated ML location estimation problem. Consider that there are a number of $m$ BSs available for locating the MS. Let $\mathbf{c}=[x, y]^{T}$ be a vector containing the Cartesian coordinate of the MS. Likewise define $\mathbf{u}_{i}=\left[x_{i}, y_{i}\right]^{T}$ to be the coordinate vector of the $i$ th BS. 
The distance between the MS and the $i$ th BS is given by

$$
d_{i}=\left\|\mathbf{c}-\mathbf{u}_{i}\right\|
$$

where $\|$.$\| stands for the 2-norm. If we assume line-of-sight$ (LOS) propagation between every $\mathrm{BS}$ and the MS, then we can measure the $d_{i}$ 's by estimating the one-way signal propagation time between the BSs and MS; i.e., time-ofarrvial (TOA) estimation [4] (BSs that exhibit non-LOS with respect to the MS may be identified and then discarded by certain algorithms [8]). Let $\mathbf{d}=\left[d_{1}, \ldots, d_{m}\right]^{T}$. The measured distance vector, denoted by $\hat{\mathbf{d}}$, can be modeled as

$$
\hat{\mathbf{d}}=\mathbf{d}+\epsilon
$$

where $\boldsymbol{\epsilon}$ represents the distance measurement error.

The mobile location problem consists in estimating $\mathbf{c}$ from the measured distance vector $\hat{\mathbf{d}}$, given knowledge of the BS locations $\mathbf{u}_{i}$. Maximum-likelihood (ML) location estimation is a probabilistic method that determines $\mathbf{c}$ by considering the probabilistic model of the error vector $\boldsymbol{\epsilon}$. In general, the exact form of the probability density function of $\epsilon$ is difficult to determine because it depends on several complicated factors such as the nonlinear structure of the TOA estimator [4]. Hence, it is common to assume [6] that $\boldsymbol{\epsilon}$ follows a Gaussian distribution with zero mean. In addition, it is reasonable to assume that each $\epsilon_{i}$ is independent of one other. Define $\boldsymbol{\Sigma}=\operatorname{diag}\left(\sigma_{1}^{2}, \ldots, \sigma_{m}^{2}\right)$ to be the covariance of $\boldsymbol{\epsilon}$. The ML location estimator is shown to be [7]

$$
\hat{\mathbf{c}}=\arg \min _{\mathbf{c} \in \mathbb{R}^{2}}(\hat{\mathbf{d}}-\mathbf{g}(\mathbf{c}))^{T} \boldsymbol{\Sigma}^{-1}(\hat{\mathbf{d}}-\mathbf{g}(\mathbf{c}))
$$

where $\mathbf{g}: \mathbb{R}^{2} \rightarrow \mathbb{R}^{m}$ has its $i$ th element given by

$$
g_{i}(\mathbf{c})=\left\|\mathbf{c}-\mathbf{u}_{i}\right\| .
$$

It is worth pointing out that (3) can be alternatively regarded as a least squares fitting problem, which serves as a reasonable source location criterion when the probability distribution of $\epsilon$ is not known.

To obtain the ML location estimate we need to solve the optimization problem in (3). Unfortunately, (3) can be shown to be a nonconvex optimization problem for which finding the globally optimal solution may not be easy. In the subsequent section, we will propose an approximation method for (3) using semidefinite relaxation.

\section{MOBILE LOCATION ESTIMATION USING SEMIDEFINITE RELAXATION}

Semidefinite relaxation (SDR) is an accurate approximation technique for a number of difficult optimization problems [10]-[14]. To facilitate the development of the SDR approximation for ML location estimation, we first focus on reformulating the (nonconvex) ML optimization problem. Then, we show how the reformulated ML problem can be approximated in a convex manner using semidefinite programming.

We should point out that the ideas behind the proposed SDR method are based on the concepts of convexity and semidefinite programming. Full details for these two topics can be found in the literature such as the introductory materials in $[15,10]$.

\subsection{Reformulation of the ML Problem}

The ML location estimation problem in (3) can be rewritten as a constrained quadratic program as follows:

$$
\begin{aligned}
\min _{\mathbf{c}, \mathbf{g}} & (\hat{\mathbf{d}}-\mathbf{g})^{T} \mathbf{\Sigma}^{-1}(\hat{\mathbf{d}}-\mathbf{g}) \\
\text { s.t. } & g_{i}^{2}=\left\|\mathbf{c}-\mathbf{u}_{i}\right\|^{2}, \quad i=1, \ldots, m \\
& g_{i} \geq 0, \quad i=1, \ldots, m
\end{aligned}
$$

Since the distance vector $\hat{\mathbf{d}}$ contains only non-negative elements, it is shown in the Appendix that (5) can be simplified as

$$
\begin{aligned}
& \min _{\mathbf{c}, \mathbf{g}}(\hat{\mathbf{d}}-\mathbf{g})^{T} \mathbf{\Sigma}^{-1}(\hat{\mathbf{d}}-\mathbf{g}) \\
& \text { s.t. } g_{i}^{2}=\left\|\mathbf{c}-\mathbf{u}_{i}\right\|^{2}, \quad i=1, \ldots, m
\end{aligned}
$$

in which the inequality constraint in $(5 \mathrm{c})$ is discarded. In the Appendix, we prove that a globally optimal solution of (6) must have $g_{i} \geq 0$ for all $i$, thus the optimal solution of (6) is that of (5). By decomposing the 2-norms in (6), the equivalent ML problem in (6) can be re-expressed as:

$$
\begin{aligned}
& \min _{\mathbf{c}, \mathbf{g}}\left[\begin{array}{ll}
\mathbf{g}^{T} & 1
\end{array}\right]\left[\begin{array}{cc}
\boldsymbol{\Sigma}^{-1} & -\boldsymbol{\Sigma}^{-1} \hat{\mathbf{d}} \\
-\hat{\mathbf{d}}^{T} \boldsymbol{\Sigma}^{-1} & \hat{\mathbf{d}}^{T} \boldsymbol{\Sigma}^{-1} \hat{\mathbf{d}}
\end{array}\right]\left[\begin{array}{l}
\mathbf{g} \\
1
\end{array}\right] \\
& \text { s.t. } g_{i}^{2}=\left[\begin{array}{ll}
\mathbf{c}^{T} & 1
\end{array}\right]\left[\begin{array}{cc}
\mathbf{I} & -\mathbf{u}_{i} \\
-\mathbf{u}_{i}^{T} & \left\|\mathbf{u}_{i}\right\|^{2}
\end{array}\right]\left[\begin{array}{l}
\mathbf{c} \\
1
\end{array}\right], i=1, \ldots, m
\end{aligned}
$$

Eq. (7) is a homogeneous quadratic program with homogeneous quadratic equality constraints. While the objective function in (7a) is convex, and the quadratic equality constraints in $(7 \mathrm{~b})$ are nonconvex. The next subsection will show how the nonconvex problem in (7) can be approximated by a semidefinite programming based convex optimization problem.

\subsection{Semidefinite Relaxation}

To illustrate the idea of semidefinite relaxation (SDR), define two matrices $\mathbf{G}=\mathbf{g g}^{T}$ and $\mathbf{C}=\mathbf{c c}^{T}$. By noting that

$$
\left[\begin{array}{l}
\mathbf{g} \\
1
\end{array}\right]\left[\begin{array}{ll}
\mathbf{g}^{T} & 1
\end{array}\right]=\left[\begin{array}{cc}
\mathbf{G} & \mathbf{g} \\
\mathbf{g}^{T} & 1
\end{array}\right],\left[\begin{array}{l}
\mathbf{c} \\
1
\end{array}\right]\left[\begin{array}{ll}
\mathbf{c}^{T} & 1
\end{array}\right]=\left[\begin{array}{cc}
\mathbf{C} & \mathbf{c} \\
\mathbf{c}^{T} & 1
\end{array}\right]
$$

and using the basic property that $\mathbf{x}^{T} \mathbf{A} \mathbf{x}=\operatorname{tr}\left\{\mathbf{x} \mathbf{x}^{T} \mathbf{A}\right\}$, the ML problem in (7) can be reformulated as

$$
\begin{gathered}
\min _{\mathbf{c}, \mathbf{g}, \mathbf{C}, \mathbf{G}} \operatorname{tr}\left\{\left[\begin{array}{cc}
\mathbf{G} & \mathbf{g} \\
\mathbf{g}^{T} & 1
\end{array}\right]\left[\begin{array}{cc}
\boldsymbol{\Sigma}^{-1} & -\boldsymbol{\Sigma}^{-1} \hat{\mathbf{d}} \\
-\hat{\mathbf{d}}^{T} \boldsymbol{\Sigma}^{-1} & \hat{\mathbf{d}}^{T} \boldsymbol{\Sigma}^{-1} \hat{\mathbf{d}}
\end{array}\right]\right\} \\
\text { s.t. } G_{i i}=\operatorname{tr}\left\{\left[\begin{array}{ll}
\mathbf{C} & \mathbf{c} \\
\mathbf{c}^{T} & 1
\end{array}\right]\left[\begin{array}{cc}
\mathbf{I} & -\mathbf{u}_{i} \\
-\mathbf{u}_{i}^{T} & \left\|\mathbf{u}_{i}\right\|^{2}
\end{array}\right]\right\}, i=1, \ldots, m \\
\mathbf{G}=\mathbf{g g}^{T}, \quad \mathbf{C}=\mathbf{c c}^{T}
\end{gathered}
$$

In the reformulated ML problem in (9), the constraints in (9b) are linear (and convex) in $(\mathbf{c}, \mathbf{C}, \mathbf{G})$ but the constraints in (9c) are nonconvex. The principle of SDR is to modify (9c) such that an approximate but convex version of (9) is obtained. From (8), the constraints in (9c) imply that the matrices

$$
\left[\begin{array}{cc}
\mathbf{G} & \mathbf{g} \\
\mathbf{g}^{T} & 1
\end{array}\right] \text { and } \quad\left[\begin{array}{cc}
\mathbf{C} & \mathbf{c} \\
\mathbf{c}^{T} & 1
\end{array}\right]
$$


are of rank 1 and symmetric positive semidefinite (PSD). If the constraints in (9c) are relaxed such that we only require the matrices in (10) to be symmetric PSD, then we obtain the following semidefinite relaxed ML problem:

$$
\begin{gathered}
\underset{\mathbf{c}, \mathbf{g}, \mathbf{C}, \mathbf{G}}{\min } \operatorname{tr}\left\{\left[\begin{array}{ll}
\mathbf{G} & \mathbf{g} \\
\mathbf{g}^{T} & 1
\end{array}\right]\left[\begin{array}{cc}
\boldsymbol{\Sigma}^{-1} & -\boldsymbol{\Sigma}^{-1} \hat{\mathbf{d}} \\
-\hat{\mathbf{d}}^{T} \boldsymbol{\Sigma}^{-1} & \hat{\mathbf{d}}^{T} \boldsymbol{\Sigma}^{-1} \hat{\mathbf{d}}
\end{array}\right]\right\} \\
\text { s.t. } G_{i i}=\operatorname{tr}\left\{\left[\begin{array}{ll}
\mathbf{C} & \mathbf{c} \\
\mathbf{c}^{T} & 1
\end{array}\right]\left[\begin{array}{cc}
\mathbf{I} & -\mathbf{u}_{i} \\
-\mathbf{u}_{i}^{T} & \left\|\mathbf{u}_{i}\right\|^{2}
\end{array}\right]\right\}, i=1, \ldots, m \\
{\left[\begin{array}{cc}
\mathbf{G} & \mathbf{g} \\
\mathbf{g}^{T} & 1
\end{array}\right] \succeq \mathbf{0}, \quad\left[\begin{array}{cc}
\mathbf{C} & \mathbf{c} \\
\mathbf{c}^{T} & 1
\end{array}\right] \succeq \mathbf{0}}
\end{gathered}
$$

where the notation $\mathbf{X} \succeq \mathbf{0}$ means that $\mathbf{X}$ is symmetric PSD The PSD constraints in (11c) are convex [10]. As (11a) and (11b) are also convex, (11) is a convex optimization problem. In the optimization literature, (11) is called a semidefinite program (SDP) [10] for which there are readily available algorithms for finding the globally optimal SDP solution; e.g., the SEDUMi package [16].

Once the optimal solution of (11), denoted by $(\hat{\mathbf{c}}, \hat{\mathbf{g}}, \hat{\mathbf{C}}, \hat{\mathbf{G}})$, is found, the solution $\hat{\mathbf{c}}$ is taken as the SDR approximate ML location estimate.

\section{SIMULATION RESULTS}

We conduct several computer simulations to evaluate the location accuracy of the proposed SDR location estimator. Two commonly used location estimator, namely the sphere interpolation (SI) estimator [9] and the linearized least squares (LLS) estimator [7] are also tested for reference. It should be mentioned that SI is a closed-form method, and that LLS is an iterative method requiring initialization. In the following simulations, the LLS estimator is initialized by the SI location estimate.

In the first simulation example, $5 \mathrm{BSs}$ are used to locate the MS. The BS locations are $[0,0] \mathrm{m},[3000 \sqrt{3}, 3000] \mathrm{m}$ $[0,6000] \mathrm{m}, \quad[-3000 \sqrt{3}, 3000] \mathrm{m}$ and $[-3000 \sqrt{3},-3000] \mathrm{m}$. The MS location is $[1000,2000] \mathrm{m}$. The TOA measurement error variances are assumed uniform; i.e., $\sigma_{1}^{2}=\sigma_{2}^{2}=\ldots=$ $\sigma_{m}^{2}$. Fig. 1 plots the mean square position errors (MSPEs) of the three location estimators against $\sigma_{i}^{2}$. The figure shows that the MSPE of the proposed SDR estimator is close to the Cramér-Rao lower bound (CRLB) for a wide range of measurement error levels. Moreover, the SI and LLS estimators exhibit the threshold effect under high measurement error levels. Such an undesirable effect does not happen in SDR location estimation.

In the second simulation example, we randomize the MS location to evaluate MSPE performance averaged over various MS locations. At each simulation trial, the MS location is randomly selected on a circle centered at the origin with radius $1000 \sqrt{5} \mathrm{~m}$. The other simulation settings are the same as those for the previous example. The results, shown in Fig. 2, are similar to those of the previous example.

In the last simulation example, the number of BSs for mobile positioning is reduced to 3 . The BS locations are $[0,0] \mathrm{m},[3000 \sqrt{3}, 3000] \mathrm{m}$ and $[-3000 \sqrt{3}, 3000] \mathrm{m}$. The MS location is $[100,-500] \mathrm{m}$. TOA measurement error variances are again uniform. The simulation results are shown in
Fig. 3. Comparing Fig. 3 with the 5-BS performance results in Fig. 1, we notice that the location performance of the SI and LLS estimators deteriorates with less BSs. In particular, the threshold effects of the two estimators happen with smaller measurement error level when less BSs are available. Like the previous results, the MSPE performance of the SDR estimator remains close to the CRLB.

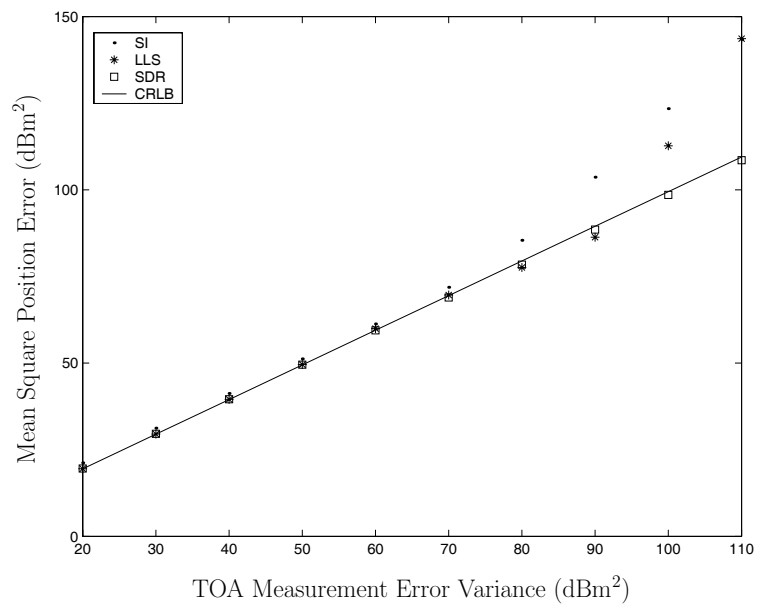

Figure 1: Location accuracy in a 5-BS system.

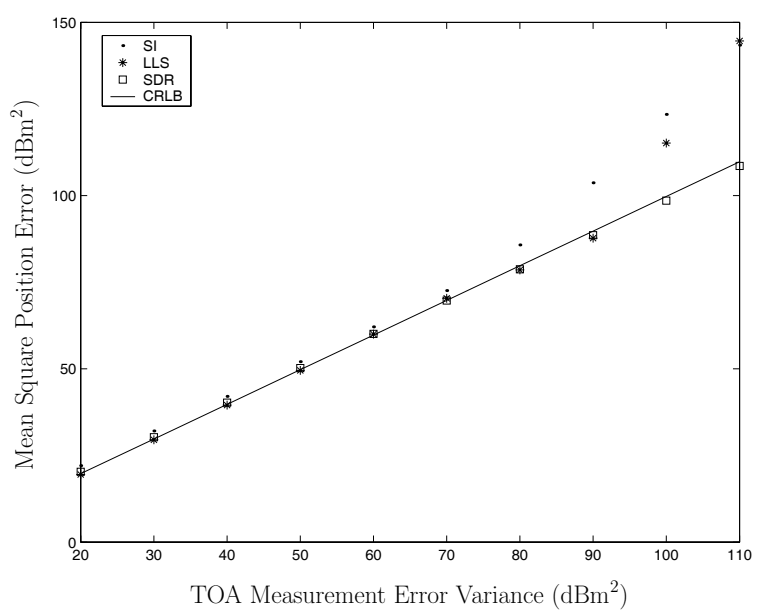

Figure 2: Location accuracy in a 5-BS system with random MS location.

\section{CONCLUSION}

The obstacle of using TOA-based ML location estimation lies in the nonconvex nature of the ML problem. We have shown in this paper how the ML problem can be approximated by a convex optimization based method called semidefinite relaxation. Simulation results have illustrated 


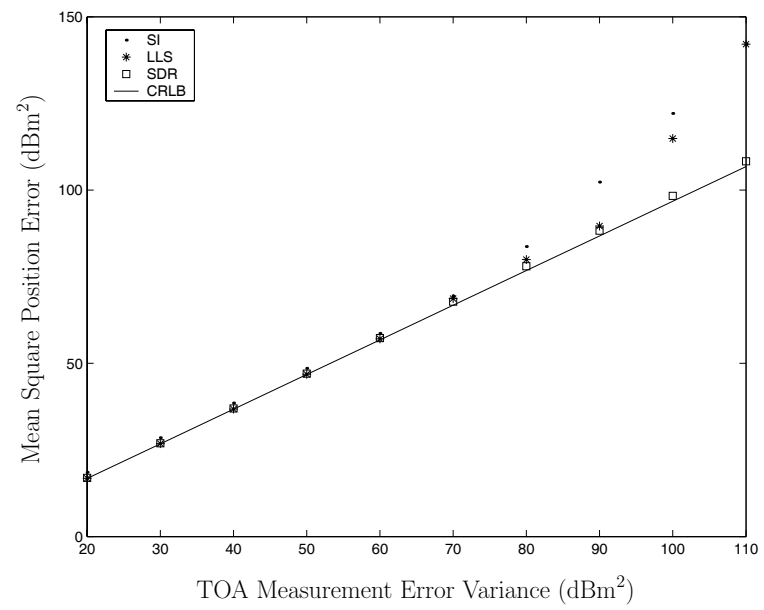

Figure 3: Location accuracy in a 3-BS system.

that the semidefinite relaxation location estimator provides an accurate approximation to the true ML.

\section{APPENDIX}

To prove the equivalence of (5) and (6), it suffices to show a globally optimal solution of $(6)$, denoted by $\left(\mathbf{c}^{\star}, \mathbf{g}^{\star}\right)$, always has $g_{i}^{\star} \geq 0$ for all $i$. The minimal objective function value in (6) can be expressed as

$$
\begin{aligned}
\left(\hat{\mathbf{d}}-\mathbf{g}^{\star}\right)^{T} \boldsymbol{\Sigma}^{-1}\left(\hat{\mathbf{d}}-\mathbf{g}^{\star}\right) & =\sum_{i=1}^{m} \frac{1}{\sigma_{i}^{2}}\left(\hat{d}_{i}-g_{i}^{\star}\right)^{2} \\
& \geq \sum_{i=1}^{m} \frac{1}{\sigma_{i}^{2}}\left(\left|\hat{d}_{i}\right|-\left|g_{i}^{\star}\right|\right)^{2} \\
& \equiv\left(\hat{\mathbf{d}}-\operatorname{abs}\left(\mathbf{g}^{\star}\right)\right)^{T} \boldsymbol{\Sigma}^{-1}\left(\hat{\mathbf{d}}-\operatorname{abs}\left(\mathbf{g}^{\star}\right)\right)
\end{aligned}
$$

where abs : $\mathbb{R}^{m} \rightarrow \mathbb{R}^{m}$ is the elementwise absolute function, and $(12 \mathrm{~b})$ is due to the fact that $\hat{d}_{i} \geq 0$ for all $i$. Eq. (12) implies that the minimal objective function value is less than or equal to that achieved by the feasible point $\left(\mathbf{c}^{\star}, \operatorname{abs}\left(\mathbf{g}^{\star}\right)\right)$. This further implies that if strict inequality in (12a) holds, then $\left(\mathbf{c}^{\star}, \mathbf{g}^{\star}\right)$ is not globally optimal. Since equality in (12a) holds if and only if $\mathbf{g}^{\star}=\operatorname{abs}\left(\mathbf{g}^{\star}\right)$, the global optimal solution must satisfy $g_{i}^{\star} \geq 0$ for all $i$.

\section{REFERENCES}

[1] CC Docket No. 94-102, "Revision of the Commissions Rules to Ensure Compatibility with Enhanced 911 Emergency Calling Systems, RM-8143," July 26, 1996.

[2] H. Koshima and J. Hosen, "Personal locator services emerge," IEEE Spectrum, pp. 41-48, Feb. 2000.

[3] J.Warrior, E.McHenry and K.McGee, "They know where you are," IEEE Spectrum, pp. 20-25, July 2003.

[4] J.J. Caffery, Jr., Wireless Location in CDMA Cellular Radio Systems, Boston: Kluwer Academic, 2000.
[5] L. Cong and W. Zhuang, "Hybrid TDOA/AOA mobile user location for wideband CDMA cellular systems," IEEE Trans. Wireless Communications, vol. 1, pp. 439447, July 2002.

[6] M.A. Sprito, "On the accuracy of cellular mobile station location estimation," IEEE Trans. Veh. Technol., vol. 50, pp. 674-685, May 2001.

[7] D.J. Torrieri, "Statistical theory of passive location systems," IEEE Trans. on Aerospace and Electronic Systems, vol. 20, pp. 183-197, March 1984.

[8] S. Al-Jazzar, J. Caffery, Jr. and H.R. You, "A scattering model based approach to NLOS mitigation in TOA location systems," Proc. IEEE 55th Vehicular Technology Conference, vol. 2, pp. 861-865, Spring 2002.

[9] J.O. Smith and J.S. Abel, "Closed-form least-squares source location estimation from range-difference measurements," IEEE Trans. Acoust., Speech, Signal Processing, vol. 35, pp. 1661-1669, Dec. 1987.

[10] L. Vandenberghe and S. Boyd, "Semidefinite programming," SIAM Rev., vol. 38, pp. 49-95, 1996.

[11] Y.E. Nesterov, "Semidefinite relaxation and nonconvex quadratic optimization," Optim. Methods Softw., vol. 9, pp. 140-160, 1998.

[12] Y. Ye, "Approximating quadratic programming with bound and quadratic constraints," Math. Program., vol. 84, pp. 219-226, 1999.

[13] W.-K. Ma, T.N. Davidson, K.M. Wong, Z.-Q. Luo and P.C. Ching, "Quasi-maximum-likelihood multiuser detection using semi-definite relaxation with applications to synchronous CDMA," IEEE Trans. Signal Processing, vol. 50, no. 4, pp. 912-922, 2002.

[14] W.-S. Lu, "Design of FIR filters with discrete coefficients: a semidefinite programming relaxation approach," 2001 IEEE Intl. Symp. Circuits Syst., vol. 2, pp. 297-300, 2001.

[15] S. Boyd and L. Vandenberghe, Convex Optimization, Department of Electrical Engineering, Stanford University, 2000. Available at: http://www.stanford. edu/ boyd/cvxbook.html.

[16] J.F. Sturm, "Using SeDuMi 1.02, a Matlab toolbox for optimization over symmetric cones," Optim. Methods Softw., vol. 11-12, pp. 625-653, 1999. Also : http: //fewcal.kub.nl/sturm/software/sedumi.html 